\title{
Tomo Direct to Deliver Static Angle Tomotherapy Treatments
}

\section{Pierfrancesco Franco ${ }^{1 *}$ and Umberto Ricardi ${ }^{2}$}

${ }^{1}$ Radiation Oncology Department, Tomotherapy Unit, Ospedale Regionale 'U. Parini', AUSL Valle d'Aosta, Aosta, Italy

${ }^{2}$ Oncology Departments, Radiation Oncology Unit, University of Torino, Turin, Italy

Radiation Therapy (RT) is a mainstay option for human cancer treatment, as it is employed as single agent or within combined modality approaches, with different timelines and aims (radical, neoadjuvant, adjuvant, palliation) and in almost all anatomic districts [1]. In recent years, significant developments have been introduced in RT planning a delivery, leading to improvements in terms of tumor control and normal tissue avoidance [2]. The Tomotherapy Hi-Art II system (Accuray Inc. , Sunnyvale, CA) is a RT platform able to deliver highly conformal intensity modulated radiotherapy (IMRT) plans within a helical geometry under image guidance (IGRT) [3]. A recent platform upgrade is named TomoDirect and it has been introduced in clinical practice in 2010 , as a suitable solution for specific clinical context characterized by particular spatial relationships between target volumes (TVs) and organs at risk (OARs) allowing for beam arrangements constrained to a limited number of directions [4]. Tomotherapy delivers 6MV-IMRT through a rotating fan beam carved by a binary multileaf collimator (MLC), since the ring gantry rotates around the treatment couch as it progresses within the gantry bore across the delivery plane [4]. Beam modulation is obtained by a single row of binary leaves for each projected angle over a rotational interval of 7 degrees, up to a total of 51 projections for each gantry revolution. This approach provides robust conformality, abrupt dose fall off and reliable accuracy. However, since radiation is delivered all around the patient, a larger integral dose might become an issue, with low dose delivery (the so called 'low dose bath') to body segments that would only receive scatter radiation with a $3 \mathrm{D}$ conformal RT approach. During the optimization process, virtual structures named 'blocks' can improve dosimetry precluding radiation from specific volumes Thus, RT delivery is limited to fewer directions with a slender set of beamlets. Since the gantry rotational velocity remains constant, while the number of treatment directions decreases (with more gantry rotations needed to deliver the prescribed dose), this might result in unnecessary prolonged treatment time. Whenever the desired treatment is constrained to a limited number of incoming directions, this planning modality might represent an issue. TomoDirect (TD) combines static gantry positions, concomitant couch translation (along the craniocaudal direction) and MLC modulation. Specifically, while the patient is translated along the superior-inferior direction past the fixed fan beam path during delivery of each treatment field (defined as a fixed gantry angle and jaw width), the beam intensity is modulated by the binary collimator while the pitch (fraction of the jaw opening advanced by the treatment couch per evolution) regulates the degree of modulation in the cranio-caudal direction. Soon after the patient has been treated from one angle, the gantry is rotated to a different beam direction and the patient is again passed through the bore for delivery of the subsequent field. To generate TD plans, the treatment field width, pitch (the TD pitch is defined as the distance of couch travel in centimetres per sinogram projection) and modulation factor represent the metrics parameter to be selected. Subsequently, the dose distribution for each beamlet contributing to TV dose is calculated by a convolution/superposition algorithm. Once the beamlet calculation step is completed, the optimization process begins and an iterative least-squares minimization method is used to optimize the objective function. During the final dose computation the optimized sinogram is converted to the delivery sinogram, accounting for leaf fluence output factors and latency data. A fine calculation grid is usually used both in the optimization and calculation process. The treatment planning software is driven by dose-based objectives, correlated penalties and region of interest-based weighting factors. For TVs, minimum and maximum dose values and corresponding penalties are used in addition to a DVH-based prescription point. OARs objectives are described by maximum dose, DVH-based constraints and related penalties. Quality assurance methods for TD treatment include 2D dose distribution verification or diode arrays-based approaches [5]. TD has been evaluated within dosimetric comparison studies, mainly in the context of breast cancer radiotherapy (whole breast radiation after breast conserving surgery, partial breast irradiation, chest wall and nodal irradiation), but also in Hodgkin's disease [6-9]. These studies provided sufficient evidence on the physical reliability of the system in the investigated scenarios. Furthermore, a preliminary clinical experience reported on TD implementation for whole breast radiation, segmental bone pain palliation and whole brain radiotherapy [4]. More mature clinical series are needed to validate the static angle tomotherapy approach; however TD seems to be an efficient, reliable and throughput oriented method to be used in selected clinical contexts.

\section{References}

1. Ricardi U, Filippi AR, Guarneri A, Giglioli FR, Ciammella P, et al. (2010) Stereotactic body radiation therapy for early stage non-small cell lung cancer: results of a prospective trial. Lung Cancer 68: 72-77.

2. Munoz F, Fiandra C, Franco P, Guarneri A, Ciammella P, et al. (2012) Tracking target position variability using intraprostatic fiducial markers and electronic portal imaging in prostate cancer radiotherapy. Radiol Med 117: 1057-1070.

3. Franco P, Numico G, Migliaccio F, Catuzzo P, Cante D, et al. (2012) Head and neck region consolidation radiotherapy and prophylactic cranial irradiation with hippocampal avoidance delivered with helical tomotherapy after induction chemotherapy for non-sinonasal neuroendocrine carcinoma of the upper airways. Radiat Oncol 7: 21.

4. Franco P, Catuzzo P, Cante D, La Porta MR, Sciacero P, et al. (2011) TomoDirect: an efficient means to deliver radiation at static angles with tomotherapy. Tumori 97: 498-502.

5. Catuzzo P, Zenone F, Aimonetto S, Peruzzo A, Casanova Borca V, et al. (2012) Technical note: patient-specific quality assurance methods for TomoDirect(TM) whole breast treatment delivery. Med Phys 39: 4073-4078.

*Corresponding author: Pierfrancesco Franco, Radiation Oncology Department, Tomotherapy Unit, Ospedale Regionale 'U. Parini', AUSL Valle d'Aosta, Viale Ginevra 3, 11100, Aosta, Italy,Tel: +390-165543420; Fax: +390165543421; E-mail: pfranco@ausl.vda.it

Received November 23, 2012; Accepted November 25, 2012; Published November 30, 2012

Citation: Franco P, Ricardi U (2012) Tomo Direct to Deliver Static Angle Tomotherapy Treatments. J Nucl Med Radiat Ther 3:e107. doi:10.4172/21559619.1000e107

Copyright: $\odot 2012$ Franco $P$, et al. This is an open-access article distributed under the terms of the Creative Commons Attribution License, which permits unrestricted use, distribution, and reproduction in any medium, provided the original author and source are credited. 
Citation: Franco P, Ricardi U (2012) Tomo Direct to Deliver Static Angle Tomotherapy Treatments. J Nucl Med Radiat Ther 3:e107. doi:10.4172/21559619.1000e107

Page 2 of 2

6. Reynders $T$, Tournel K, De Coninck P, Heymann S, Vinh-Hung V, et al. (2009) Dosimetric assessment of static and helical tomotherapy in the clinical implementation of breast cancer treatments. Radiother Oncol 93: 71-79.

7. Schubert LK, Gondi V, Sengbusch E, Westerly DC, Soisson ET, et al. (2011) Dosimetric comparison of left-sided whole breast irradiation with 3DCRT, forward-planned IMRT, inverse-planned IMRT, helical tomotherapy, and topotherapy. Radiother Oncol 100: 241-246.
8. Jones R, Yang W, Read P, Sheng K (2011) Radiation therapy of postmastectomy patients with positive nodes using fixed beam tomotherapy. Radiother Oncol 100: 247-252.

9. Fiandra C, Filippi AR, Catuzzo P, Botticella A, Ciammella P, et al. (2012) Different IMRT solutions vs. 3D-conformal radiotherapy in early stage Hodgkin's lymphoma: dosimetric comparison and clinical considerations. Radiat Oncol 7: 186. 